\title{
Ueber die Ursachen der Gewichtsveränderungen Neugeborener.
}

\author{
Von \\ F. A. $K \in h r \in r$.
}

In verschiedenen Gebäranstalten Deutschlands hat man die neugeborenen Kinder bis zu ihrer Entlassung täglich gewogen und dabei die zuerst von Chaussier gemachte Beobachtung bestätigt ${ }^{1}$ ), dass dieselben in den ersten Lebenstagen an Körpergewicht nie zunehmen, sondern im Gegentheil abnehmen, nach Verlauf von 2-6 Tagen in der Mehrzahl der Fälle den Verlust wieder zu ersetzen anfangen, in einer Minderzahl aber bis zum Tode oder der Aufbesserung ihrer Ernährungsverhältnisse zu atrophiren fortfahren.

In Haake's Wägungen betrug das Minimum der Gewichtsabnahme innerhalb der ersten 24 Stunden 1 Zolloth, das Maximum 17 Loth, im Durchschnitt 8 Loth, bei Winckel Minimum 2, Maximum 16, Durchschnitt 6,95 Loth bei Knaben, 81/2 Loth bei Mädchen. Die totale Gewichtsabnahme in den ersten Tagen ergab durchschnittlich (nach $\mathrm{Haake}$ ) bei Knaben 12,2 Loth oder 1/1 6 -1/17 des anfänglichen Körpergewichtes, bei Mädchen 12,44 Loth oder $1 / 15-1 / 16$ des primären Gewichtes; nach Winckel 14,51 Loth im Ganzen, und zwar bei Knaben 12,4,

1) Siehe Quetelet, ,Ueber'd. Menschen“. Uebers. von Riecke, 1838. S. 357. Bartsch, in "Beobachtungen über den Stoffwechsel Neugeborener". Marburger Dissertation, 1859. Breslau, in den Denkschrift. d. med.-chirur. Ges. d. Cantons Zürich. E. v. Si ebold in der "Monatsschrift f. Geburtsk." 15. 337. Haake, ebendas., 19, 339. Winckel, ebendas., 19, 416. 
bei Mädchen 13,5 Loth. Die Schwankungen der Abnahme bewegten sich zwischen 6 und 30 Loth. Am neunten Tage nach der Geburt hatten $33 \%$ der Kinder und zwar $37 \%$ der Knaben, $41 \%$ der Mädchen ihr Geburtsgewicht noch nicht wieder erreicht (4\% nach einer rückfälligen Abnahme), $9 \%$ waren gleich schwer wie bei der Geburt, $58 \%$ hatten an Gewicht zugenommen und zwar $30 \%$ derselben vom 2., $58 \%$ vom $3 ., 22 \%$ von dem 3-6. Tage an (Haake). In $3 / 4$ der ausgetragenen, gesunden und mit Muttermilch ernährten Kinder fand Winckel schon am 3-4 Tage eine Zunahme, die, auf das niedrigste beobachtete Gewicht bezogen, in den ersten zehn Tagen durchschnittlich 15,5 Loth betrug, bei Knaben 16,4, bei Mädchen 14,1 Loth. Die mit Kuhmilch aufgefütterten Kinder waren am zehnten Tage noch leichter als zur Zeit der Geburt und keines zeigte noch eine beginnende Zunahme. Dasselbe gilt für frühgeborene Kinder.

Nach den Ergebnissen dieser Wägungen hielt man sich von der Gewichtsabnahme der Neugeborenen in den ersten Tagen als einer physiologischen Thatsache überzeugt und bezog dieselbe auf ein Missverhältniss zwischen den Einnahmen und Ausgaben des kindlichen Organismus, der eine bedeutende Quantität Meconium, ferner Urin, Respirations- und Perspirationsgase abgiebt und nur spärliche Nahrung aufnimmt, welche überdem in Folge der chemischen Eigenschaften des Colostrum zwar das Meconium abzuführen im Stande sei, aber einen nur geringen Nährwerth besitze. Das Neugeborene betrachtete man demnach einem Hungernden gleich. Genöthigt, zur Production von Wärme und Muskelkraft sowie von Secreten ein gewisses Quantum seines Körpermaterials abzugeben, ohne entsprechende Nahrungseinnahmen zu haben, muss das Kind in den ersten Lebenstagen collabiren, was sich schon der blossen Inspection durch die abnehmende Körperfülle verräth.

Diese Auffassung, dass eine Gewichtsabnahme die Regel und eine Art physiologischer Nothwendigkeit darstelle, suchte v. Rittershain in dem Jahresberichte der $k$. böhmischen Landes-Findelanstalt in Prag für 1866 (Prager Vierteljahrschrift, 25. Jahrg. 1868, Bd. 97. S. 21 ff.) zurückzuweisen. Seine Beobachtungen bezogen sich auf 144 Kinder, die zuerst bei der Aufnahme, aber wohl nur in einer Minorität der Fälle gleich nach der Geburt, und zum zweiten Male zwischen dem 3-7., durchschnittlich am fünften Tage, gewogen wurden. Bei der zweiten Wägung erschienen zwölf dieser $144 \mathrm{Kinder,} \mathrm{also} 8,3 \%$, gleich schwer wie zuerst, 
$76=52,7 \%$ hatten zugenommen, durchschnittlich um 5̆,1ö österr. Loth, $56=38,8 \%$ zeigten eine Abnahme von 5,64 Loth per Kopf. Dieses Beobachtungsmaterial hielt R. ,für schlagend genug, die Annahme eines constanten, also naturgemässen Gewichtsverlustes des Kindes innerhalb der ersten Lebenswoche als unrichtig zu erweisen".

Fassen wir diese Angaben nebst der Schlussfolgerung etwas genauer ins Auge, so ergeben sich gewisse Bedenken gegen die Ausführung der Wägungen, wie gegen die Beweiskraft der Ergebnisse. Denn einmal sind, wie $R$. selbst sagt, nicht alle Kinder gleich nach der Geburt gewogen worden. Es ist sogar in Berücksichtigung des Verhältnisses zwischen Gebär- und Findelhaus sehr wahrscheinlich, dass nur eine Minderzahl von Kindern mit ihrem wahren Geburtsgewichte notirt ist. Folglich muss, im Hinblick auf die Beobachtungen der früher genannten Forscher, R. eine Anzahl Kinder während der Periode der Gewichtsabnahme zum ersten Male gewogen haben, und wird denn in weiterer Consequenz die nachherige Zunahme scheinbar früher und eclatanter hervortreten, als bei Vornahme der ersten Wägung gleich nach der Geburt. Es ist also der Ausgangspunkt der Vergleichung keineswegs verlässlich. Ferner wurden die Wägungen nicht an jedem einzelnen Lebenstage, sondern durchschnittlich erst am fünften Tage wiederholt. Nun haben aber Hake und Winckel gezeigt, dass bei allen Kindern am ersten, bei vielen auch in den folgenden Tagen das Gewicht abnimmt. Ferner fand Haake bei $20 \%$ der mit Gewichtszunahme in den ersten noun Tagen verzeichneten Kinder vom zweiten, bei $58 \%$ vom dritten, bei $22 \%$ vom 3-6. Tage an eine Gewichtszunahme, und Winckel beobachtete bei $3 / 4$ aller zunehmenden Kinder den Anfang der Zunahme am 3-4. Tage. Rittershain hat also gerade die für den Nachweis der Gewichtsabnahme wichtigste Periode der ersten fünf Tage ausser Acht gelassen, and musste deshalb viele Kinder als zuriehmend bezeichnen, die anfänglich an Gewicht eingebüsst, dann das Verlorene ausgeglichen und sogar bis zum fünften Tage wieder neues Körpermaterial angesetzt hatten. Da endlich R. eine Anzahl zweiter Wägungen erst am 6-7. Tage anstellte, so steigt a priori die Wahrscheinlichkeit, dass er mehr Zunahmen für den (durchschnittlichen) fünften Tag gewinnen musste als andere Beobachter. Aus diesen Gründen können wir die Zahlen Rittershain's, der in $52 \%$ der Fälle am fünften Tage eine Zunahme gegenüber dem Ergebnisse der ersten 
Wägung constatirte, während z. B. Haake am fünften Tage nur $39 \%$ Zunahmen aufzählt, nicht für beweisend halten.

Betrachten wir nun, welche Factoren für die Gewichtsschwankungen, deren Eintritt und Grösse von Bedeutung sind, so hat $\mathrm{H}$ a ake angegeben, dass die secundäre Gewichtszunahme keineswegs vorzugsweise mit der Zeit des Abfallens des Nabelstranges zusammenfällt, also beide Vorgänge nichts mit einander zu thun haben, sondern, wenn überhaupt in der ersten Woche eine Zunahme erfolgt, dies häufiger zwischen dem 2-3., als zwischen dem 3-6. Tage geschieht. Winckel dagegen fand, dass die $\mathrm{Zu}-$ nahme mit dem Tage des Nabelschnurabfalles anfängt in $54 \%$, einen Tag nachher in $33 \%$, einen Tag vorher in $11 \%$. Auf Grund der noch anzuführenden Beobachtungen bei Säugethieren möchte ich einen näheren Zusammenhang beider Erscheinungen in Uebereinstimmung mit Haake für unwahrscheinlich halten.

Haake und Winckel haben ferner, den Breslau'schen Angaben entgegen, nachgewiesen; dass Knaben im Durchschnitt weniger an Körpergewicht verlieren, früher und in höheren Procenten [66\% Knaben: $46 \%$ Mädchen] an Gewicht zunehmen als Mädchen.

Breslau und Haake zeigten ausserdem, dass eine grössere Zahl $(64,1 \%)$ der von Mehrgebärenden ernährten Kinder innerhalb der ersten neun Tage zunimmt als der Kinder Erstgebärender, auf welche nưr $50 \%$ Zunahmen fallen.

Bezüglich des Verhältnisses zwischen der Grösse des ursprünglichen Körpergewichtes und der Häufigkeit der Gewichtszunahmen in den 'ersten neun Tagen ergiebt die Berechnung aus den Haake'schen Tabellen

bei 26 Kindern ron 5-6 Pfd. (excl.) eine Frequenz der Zunahmen von . . $61 \%$, eine Frequenz der Zunahmen von . . $66 \%$, eine Frequenz der Zunahmen von . . 35\%, , 4 , , 8-8 , 12 Lth. (excl.) eine Frequenz der Zunahmen von $25 \%$.

Aus dieser Tabelle würde man folgern müssen, dass die schweren Kinder später (wenn überhaupt) an Gewicht zunehmen als die leichten. Allein hier tritt die Kleinheit der Zahlen störend 
entgegen und nöthigt zu grösster Vorsicht in den Schlüssen. Wünschenswerth muss es aber sein, diese Frage durch tägliche Wägungen einer weit grösseren Zahl von Kindern zu beantworten.

Die anderen bis jetzt noch nicht in Zahlen ausgedrückten Factoren der primären Gewichtsabnahme werden weiter unten näher untersucht werden.

Man kann nicht läugnen, dass die Thatsache des anfänglichen Gewichtsverlustes der Neugeborenen eine etwas auffallende Erscheinung ist. Man fragt sich billig, ob das neugeborene Kind mit anderen Geschöpfen das Loos des Collapsus in den ersten Lebenstagen theile, oder ob das bevorzugte Menschengeschlecht in dieser Beziehung eine etwas zweifelhafte Ausnahmestellung in der Thierreihe einnehme. Ist letzteres richtig, und die folgenden Angaben werden dies bejahen, so werden wir uns fragen müssen, ob etwa ein physiologischer Unterschied in dem Eintritte oder Verlaufe der Milchabsonderung beim menschlichen Weibe und den Säugern besteht, ob vielleicht das Saugen von den Kindern und neugeborenen Säugethieren mit ungleicher Energie geübt wird, oder ob gewisse Missstände in der Mutter - oder Kinderpflege oder in beiden den Abfall des Kindesgewichtes nach sich ziehen?

Diese Fragen und Ueberlegungen bestimmten mich vor einigen Jahren eine Reihe von Wägungen bei neugeborenen Thieren anzustellen, und ist auch die Zahl der Fälle nur klein, so scheinen mir doch die gewonnenen Ergebnisse für die Frage nach der hauptsächlichen Ursache der Gewichtsabnahme nicht ohne Bedeutung zu sein. 
Folgende sind die Resultate meiner Wägungen:

\section{Hunde.}

\begin{tabular}{|c|c|c|c|c|c|c|c|}
\hline $\begin{array}{l}\text { Tag und } \\
\text { Stunde } \\
\text { der } \\
\text { Wägung }\end{array}$ & $\begin{array}{l}\text { Alter der } \\
\text { Jungen in } \\
\text { Stunden }\end{array}$ & $\begin{array}{l}\text { Gewicht der ein- } \\
\text { zelnen Jungen in } \\
\text { Grammen }\end{array}$ & 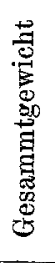 & 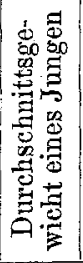 & $\begin{array}{c}\text { Schwan } \\
\text { des } \\
\text { schni } \\
\text { wich } \\
\text { Gram- } \\
\text { men }\end{array}$ & 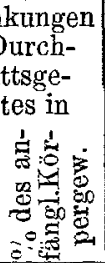 & Anmerkungen \\
\hline
\end{tabular}

Nr. 1. Pinscherhunde.

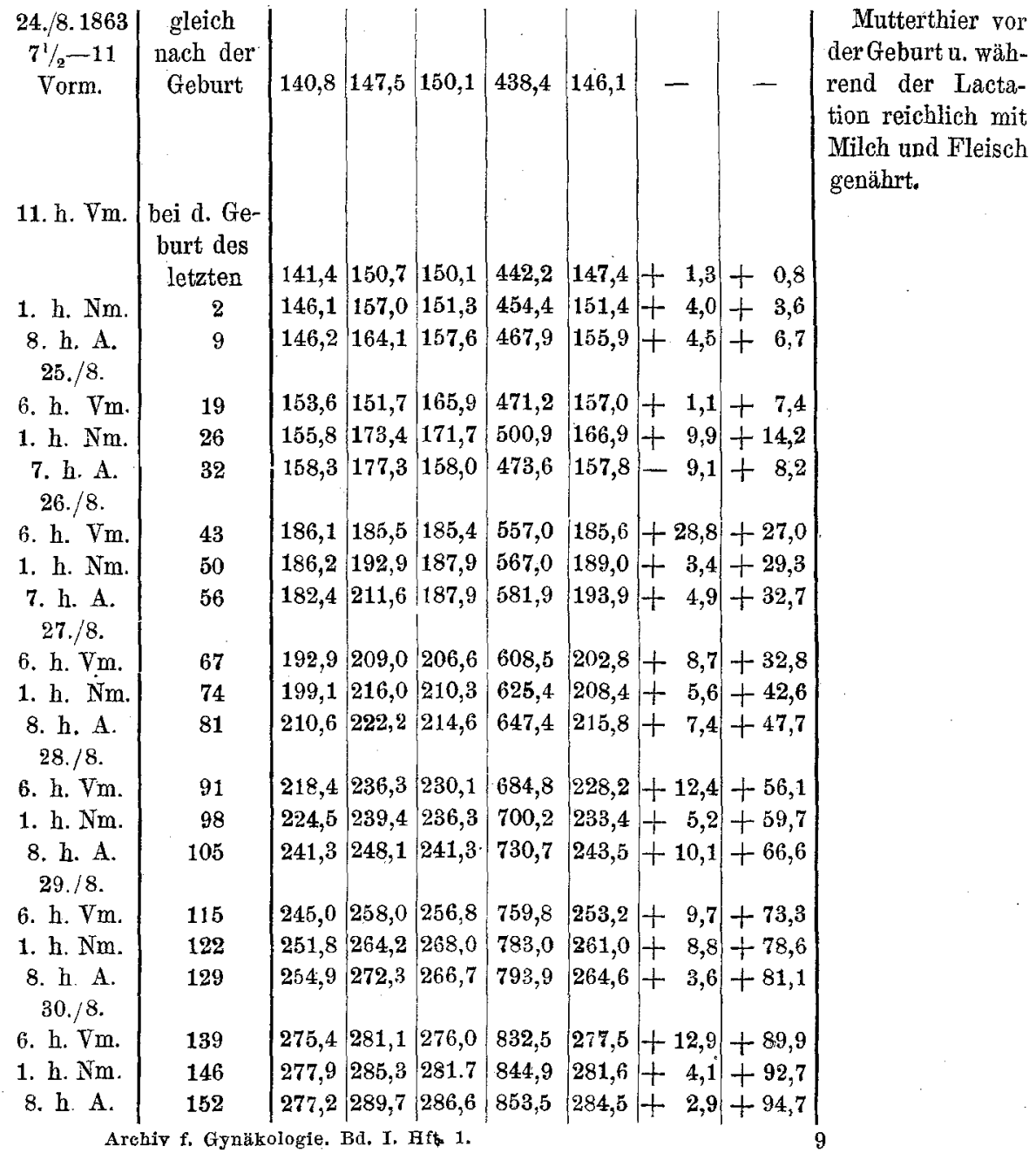




\begin{tabular}{|c|c|c|c|c|c|c|c|c|}
\hline $\begin{array}{c}\begin{array}{c}\text { Tag und } \\
\text { Stunde } \\
\text { der } \\
\text { Wägung }\end{array} \\
\\
31 . / 8 .\end{array}$ & $\begin{array}{l}\text { Alter der } \\
\text { Jungen in } \\
\text { Stunden }\end{array}$ & \multicolumn{3}{|c|}{$\begin{array}{l}\text { Gewicht der einzel- } \\
\text { nen Jungen in } \\
\text { Grammen }\end{array}$} & 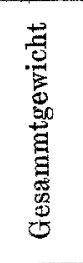 & 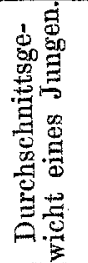 & $\begin{array}{l}\text { Schwankungen } \\
\text { des Durch- } \\
\text { schnittsgewich- } \\
\text { tes in }\end{array}$ & $\begin{array}{l}\text { nkungen } \\
\text { Durch- } \\
\text { sgewich- } \\
\text { s in } \\
\% \text { des an- } \\
\text { fänglichen } \\
\text { Körper- } \\
\text { gewichts }\end{array}$ \\
\hline $\begin{array}{l}31 . / 8 . \\
\text { 6. h. Vm. }\end{array}$ & 162 & 287,2 & 300,2 & 299,6 & 887,0 & 295,6 & $+11,1$ & $+102,3$ \\
\hline 1. h. $\mathrm{Nm}$ & 16 & 295,9 & 302,0 & 300,0 & 897,9 & 299,3 & $+3,7$ & $+104,1$ \\
\hline $\begin{array}{c}\text { 8. h. A. } \\
\text { 1. } / 9 .\end{array}$ & 176 & 300,8 & 305,2 & 302,0 & 908,0 & 302,6 & $+3,3$ & $+107,1$ \\
\hline 6. h. $\mathrm{Vm}$. & 186 & 303,9 & 312,4 & 303,0 & 919,3 & 306,4 & $+3,8$ & $+109,7$ \\
\hline 2. h. $\mathrm{Nm}$. & 19 & 317,9 & 308,3 & 304,6 & 930,8 & 310,2 & $+3,8$ & $+112,3$ \\
\hline $\begin{array}{l}\text { 8. h. A. } \\
2 . / 9 \text {. }\end{array}$ & 200 & 321,0 & 326,7 & 319,9 & 967,6 & 322,5 & $+12,3$ & $+120,7$ \\
\hline 6. h. Vm. & 21 & 332,2 & 334,7 & 324,8 & 991,7 & 330,5 & $+8,0$ & $+125,5$ \\
\hline 1. h. Nm. & 21 & 335,9 & 3484 & 324,8 & 1009,1 & 336,8 & $+5,8$ & $+130,1$ \\
\hline $\begin{array}{l}\text { 8. h. A. } \\
3 . / 9 \text {. }\end{array}$ & 224 & 340,3 & 341,6 & 333,5 & 1015,4 & 338,4 & $+2,1$ & $+131,4$ \\
\hline 6. h. Vm. & 234 & 355,2 & 349,0 & 339.7 & 1043,9 & 347,9 & $+9,5$ & $+138,1$ \\
\hline 1. h. $\mathrm{Nm}$. & 24 & 363,9 & 352,7 & 347,2 & 1063,8 & 354,6 & $+6,5$ & $+149,7$ \\
\hline $\begin{array}{l}\text { 8. h. A. } \\
4 . / 9 \text {. }\end{array}$ & 248 & 370,1 & 355,8 & 343,6 & 1069,5 & 356,5 & $+1,9$ & $+144,0$ \\
\hline 6. h. Vm. & & & 369,5 & 358,9 & 1102,4 & 367,4 & $+10,9$ & $+151,4$ \\
\hline 1. h. Nm. & 265 & 374,0 & 371,9 & 358,3 & 1104,2 & 368,0 & $+0,6$ & $+151,8$ \\
\hline $\begin{array}{l}\text { 8. h. A. } \\
\text { 5. } / 9 \text {. }\end{array}$ & & 382,1 & 374,0 & 365,7 & 1121,8 & 373,9 & $+5,9$ & $+155,9$ \\
\hline 6. h. Vm. & 282 & 395,8 & 389,6 & 374,6 & 1160,0 & 386,6 & $+12,5$ & $+164,6$ \\
\hline 1 h. $\mathrm{Nm}$. & & & & 383,3 & 1187,9 & 395,9 & $+9,3$ & $+170,9$ \\
\hline $\begin{array}{l}\text { 8. h. A. } \\
6 . / 9 \text {. }\end{array}$ & 296 & 413,1 & 398,3 & 388,9 & 1200,3 & 400,1 & $+4,2$ & $+173,8$ \\
\hline 6. h. Vm. & & & & 394,5 & & 402,5 & $+2,4$ & $+175,5$ \\
\hline 1. h. $\mathrm{Nm}$. & 313 & 426,7 & 410,6 & 395,7 & 1233,0 & 411,0 & $+8, \tilde{0}$ & $+181,3$ \\
\hline $\begin{array}{l}\text { 8. h. A. } \\
7 . / 9 .\end{array}$ & 320 & 429,9 & 413,1 & 398,8 & 1241,8 & $.413,9$ & $+2,9$ & $+183,1$ \\
\hline 6. h. Vm. & 33 & & 435,5 & 415,6 & $129 ;, 1$ & 432,0 & +18 & $+195,6$ \\
\hline 1. h. $\mathrm{Nm}$. & 38 & 446,6 & 436,7 & 418,1 & 1301,4 & 433,8 & $+1,8$ & $+196,9$ \\
\hline 8. h. A. & 343 & 455,9 & 433,6 & 424,9 & 1314,4 & 438,1 & $+4,3$ & $+199,8$ \\
\hline
\end{tabular}




\begin{tabular}{|c|c|c|c|c|c|c|c|}
\hline $\begin{array}{l}\text { Tag und } \\
\text { Stunde } \\
\text { der } \\
\text { Wägung }\end{array}$ & $\begin{array}{l}\text { Alter der } \\
\text { Jungen in } \\
\text { Stunden }\end{array}$ & $\begin{array}{l}\text { Gewicht } \\
\text { der einzelnen Jungen } \\
\text { in Grammen }\end{array}$ & 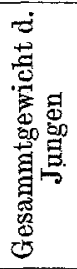 & 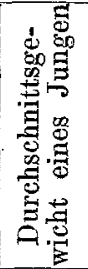 & $\begin{array}{r}\text { Schw: } \\
\text { des } \\
\text { schnitt } \\
\text { te } \\
\text { Gram- } \\
\text { men }\end{array}$ & 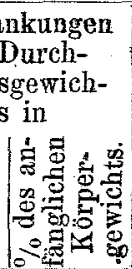 & Anmerkung. \\
\hline
\end{tabular}

Nr. 2. Pinscherhunde.

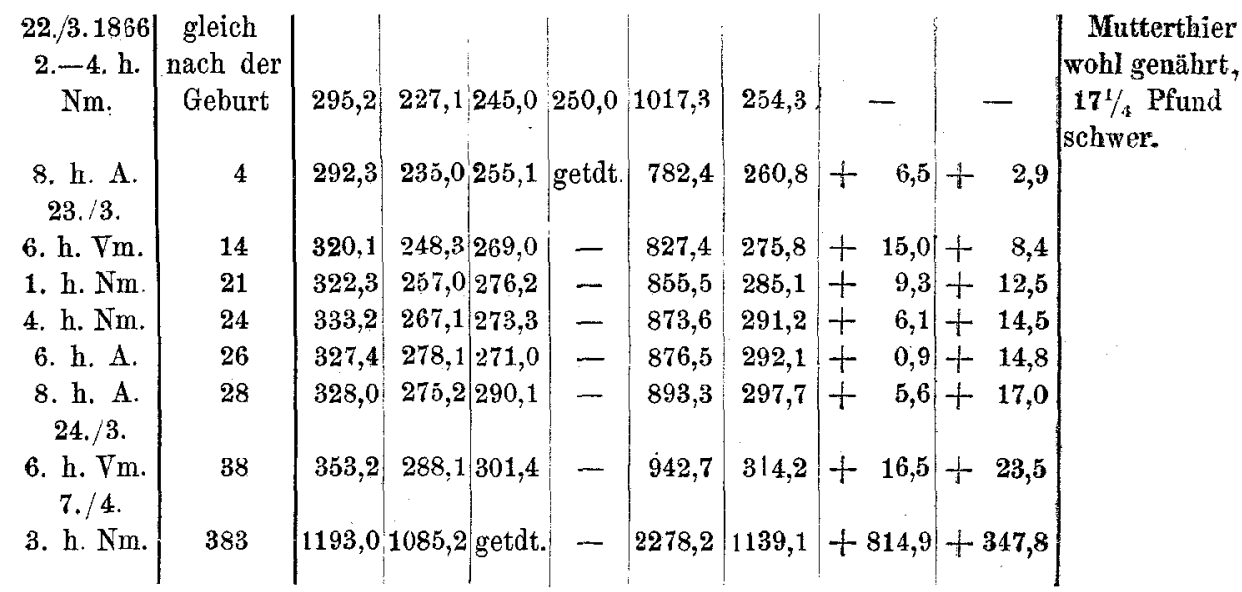

Nr. 3. Dachshunde.

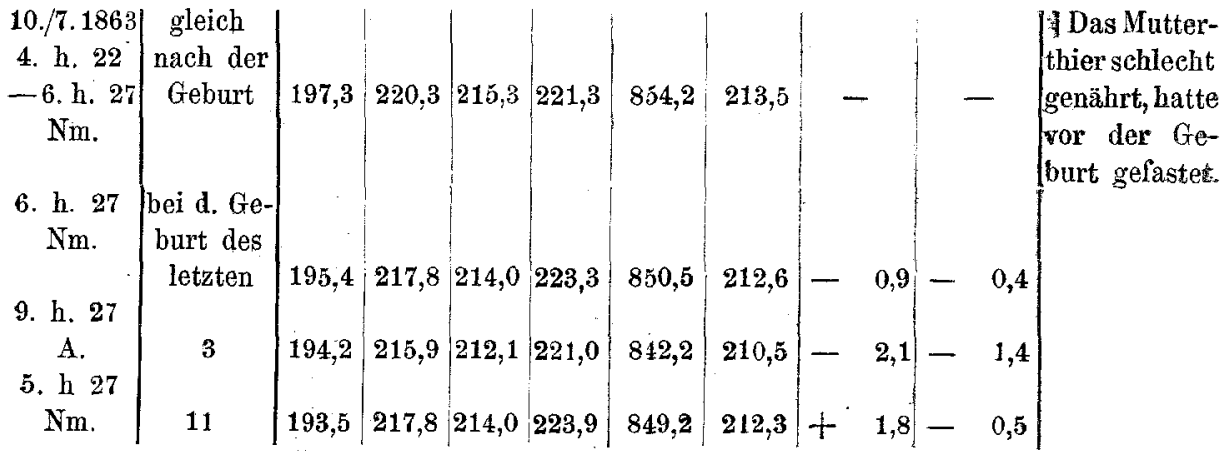




\section{Katzen.}

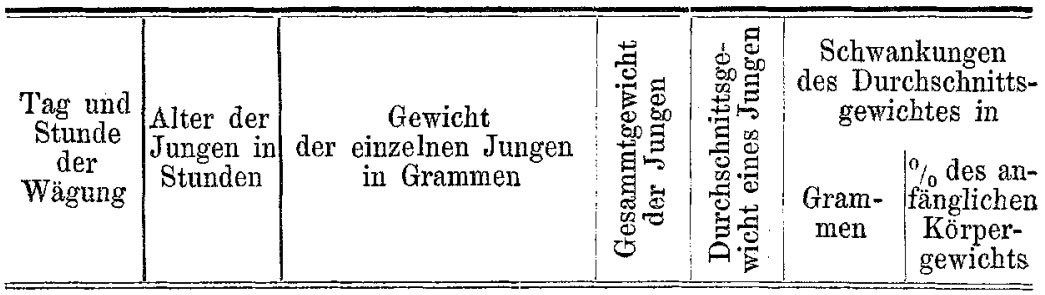

Nr. 1.

\section{6./4.1861 gleich}

9. h. Vm. nach der

Geburt d.

27./4.

9. h. $\nabla \mathrm{m}$. 28. $/ 4$.

9. h. Vm. 29./4.

9. h. Vm. $30 . / 4$

9. h. Vm. $1 . / 5$.

9. h. Vm. $2 . / 5$.

9. h. $\mathrm{Vm}$. 3./5.

9. h. Vm. 4./5.

9. h. $\mathrm{Ym}$. 5./5.

9. h. Vm. 6. $/ 5$.

9. h. Vm. 7./ 5 .

9. h. Vm. $8 . / 5$.

9. h. Vm. 9./5.

9. h. Vm. 10./5.

9. h. Vm.

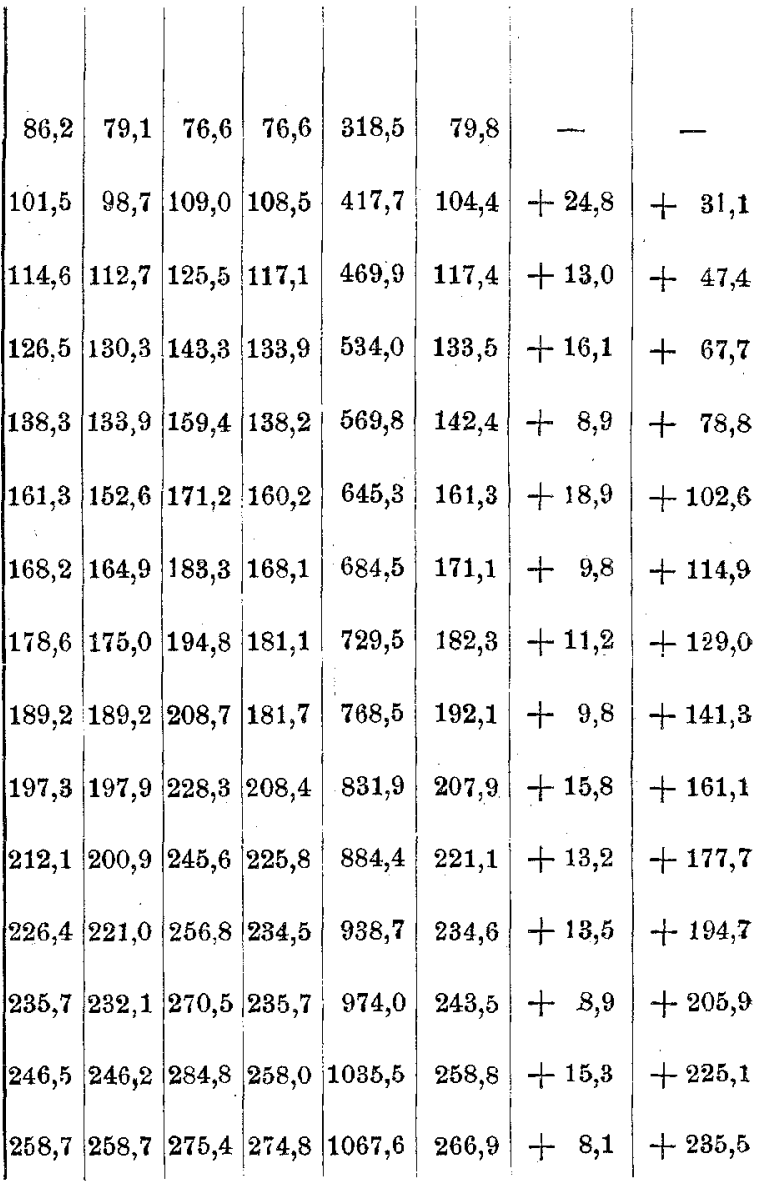




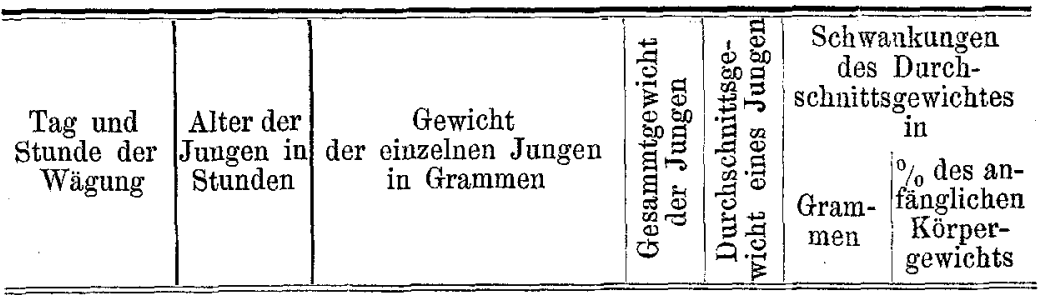

Nr. 2.

27./4. $1862 \mid$ gleich 10. h. 30. Vm. nach der Geburt d.

$28 . / 4$.

10. h. $30 . \mathrm{Vm}$. $29 . / 4$.

10. h. $30 . \mathrm{Vm}$, $30 . / 4$.

10. h. 30. Vm.

1./5.

10. h. 30. Vm. 2./5.

10. h. 30. Vm: letrten

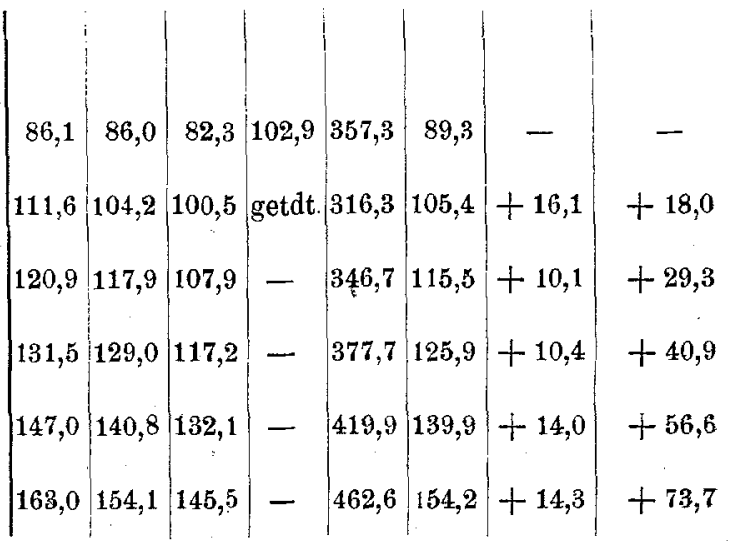

Nr. 3.

11./9. 1862 gleich

11. h. 35. A. nach der

-2.h.30. N. Geburt

12./9.

2. h. $30 . \mathrm{Nm}$.

6. h. $30 . \mathrm{Vm}$.

10. h. $30 . \mathrm{Vm}$

2. h. 30. Nm.

8. h. 30. A.

13. $/ 9$.

6. h. $30 . \mathrm{Vm}$.

14./9.

6. h. 30 . Vm.

15. $/ 9$.

6. h. 30. Vm.

8. h. 30.A.

16./9.

6. h. $30 . \mathrm{Vm}$. gleich nach der Geburt d. letzten

4 8

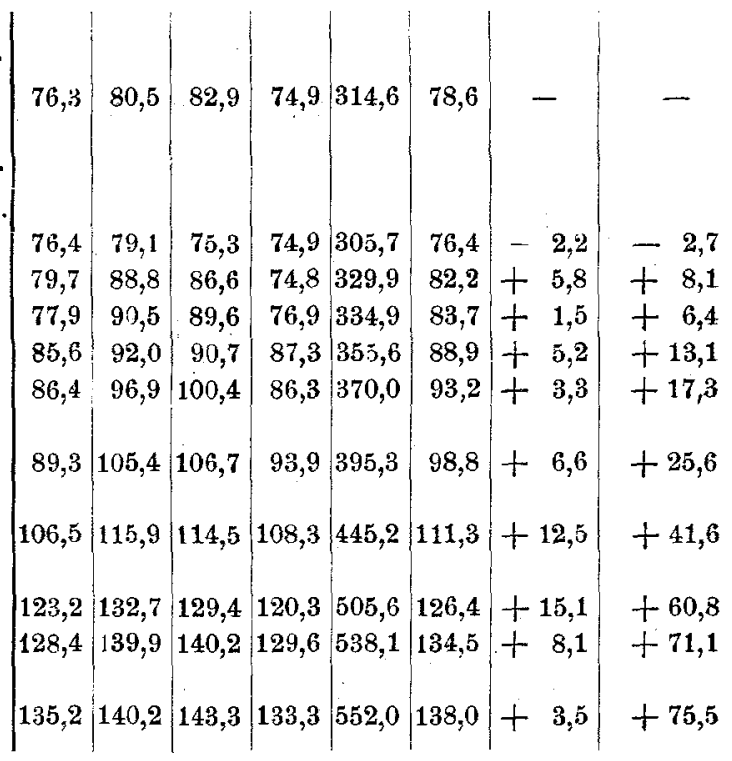




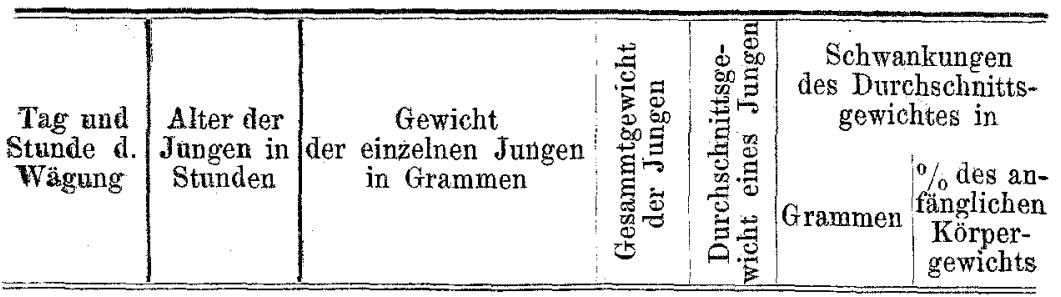

Nr. 4.

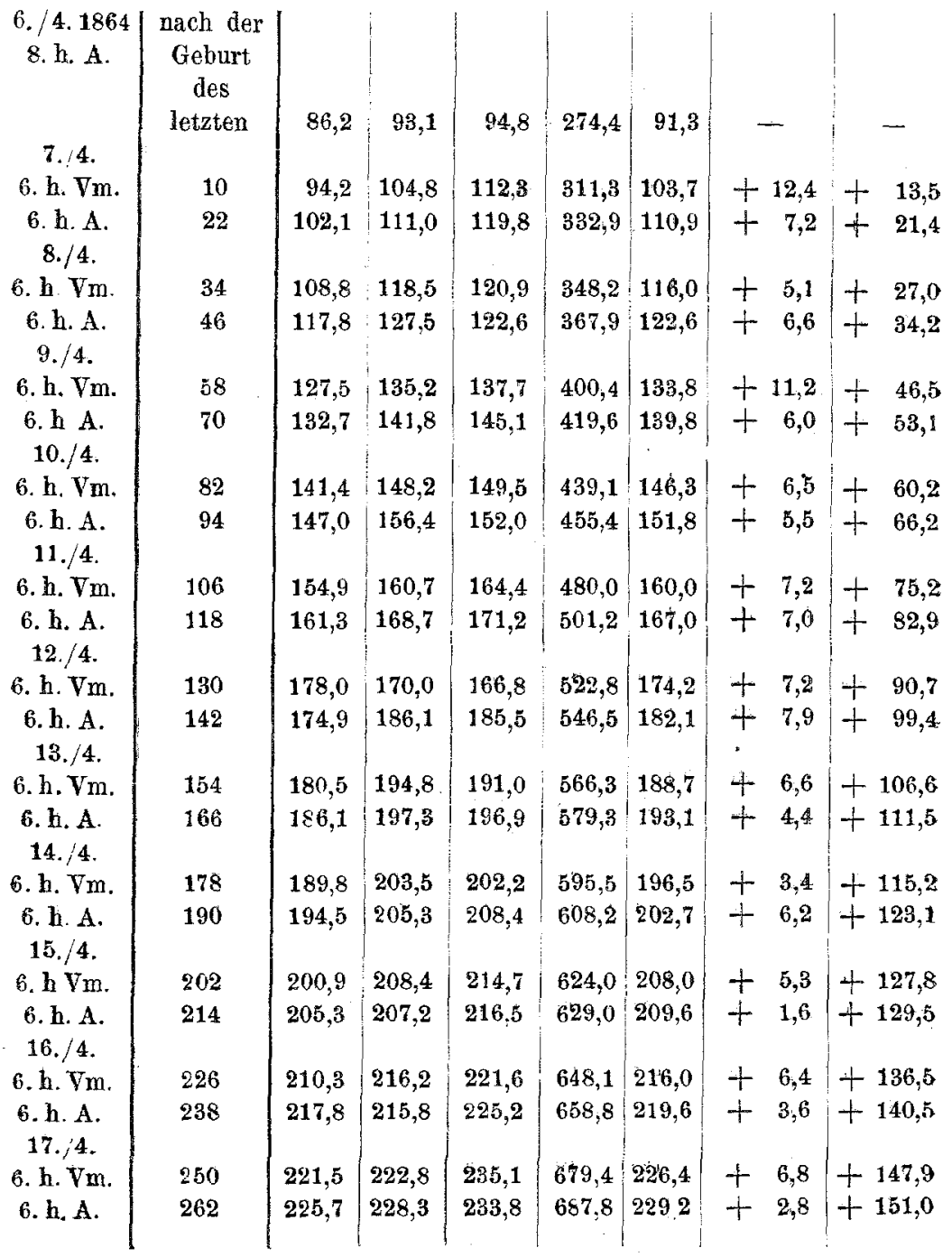




\begin{tabular}{|c|c|c|c|c|c|c|c|c|c|}
\hline $\begin{array}{l}\text { Tag und } \\
\text { Stunde d. } \\
\text { Wägung }\end{array}$ & $\begin{array}{l}\text { Alter der } \\
\text { Jungen in } \\
\text { Stunden }\end{array}$ & \multicolumn{4}{|c|}{$\begin{array}{l}\text { Gewicht } \\
\text { der einzelnen Jungen } \\
\text { in Grammen }\end{array}$} & 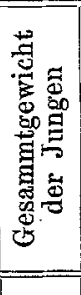 & 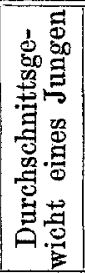 & $\begin{array}{r}\text { Schwar } \\
\text { des Durc } \\
\text { gewic } \\
\text { Grammen }\end{array}$ & $\begin{array}{l}\text { nkungen } \\
\text { chschnitts- } \\
\text { htes in } \\
\text { o\% des an- } \\
\text { fänglichen } \\
\text { Körper- } \\
\text { gewichts }\end{array}$ \\
\hline $\begin{array}{c}18 . / 4 \\
6 . \mathrm{h} . \mathrm{Vm}\end{array}$ & 274 & 241,3 & 230,7 & 234,5 & - & 706,4 & 235,4 & $+6,2$ & $+157,8$ \\
\hline $\begin{array}{c}\text { 6. h. A } \\
19 . / 4\end{array}$ & 286 & 240,0 & 235,7 & 238,2 & - & 713,9 & 237,9 & $+2,5$ & $+160,5$ \\
\hline 6. h. Vm. & 298 & 245,0 & 240,7 & 242,5 & - & 728,2 & 242,7 & $+4,8$ & $+165,8$ \\
\hline $\begin{array}{c}\text { 6. h. A. } \\
20 . / 4 \text {. }\end{array}$ & 310 & 243,1 & 245,6 & 253,1 & - & 741,8 & 247,2 & $+4,5$ & $+170,7$ \\
\hline 6. h. Vm. & 322 & 249,4 & 253,1 & 260,5 & - & 763,0 & 254,3 & $+7,1$ & $+170,7$ \\
\hline 6. h. A. & 334 & 254,9 & 260,5 & 260,6 & - & 776,0 & 258,6 & $+4,3$ & +183.2 \\
\hline
\end{tabular}

Nr. 5.

\begin{tabular}{|c|c|c|c|c|c|c|c|c|c|}
\hline \multirow{4}{*}{$\begin{array}{l}\text { 7./5. } 1867 \\
\text { 3. h. } 37 .- \\
\text { 7.h. Nm. } \\
\text { 7.h. A. }\end{array}$} & gleich & & & & & & & & \\
\hline & nach der & & & & & & & & \\
\hline & $\begin{array}{l}\text { Geburt } \\
\text { nach der }\end{array}$ & 88,3 & 97,5 & 80,5 & 94,4 & 360,7 & 90,1 & - & - \\
\hline & $\begin{array}{c}\text { Geburt d. } \\
\text { letzten }\end{array}$ & 89,9 & 100,7 & 77,6 & 94,4 & 362,6 & 90,6 & $+0,5$ & + \\
\hline $\begin{array}{c}\text { 9.h. A. } \\
8 . / 7 .\end{array}$ & 2 & 93,7 & 105,5 & 82,9 & 96,9 & 379,0 & 94,7 & $+4,1$ & + \\
\hline 6. h. $\mathrm{Vm}$. & 11 & 99,8 & 117,6 & 92,4 & 99,8 & 409,6 & 102,4 & $+8,7$ & $+13,6$ \\
\hline $\begin{array}{c}\text { 6. h. A. } \\
9 . / 7 \text {. }\end{array}$ & 23 & 105,7 & 122,2 & 98,7 & 102,6 & 429,2 & 107,3 & $+4,9$ & $+19,1$ \\
\hline 6. h. Vm. & 35 & 115,9 & 130,6 & 104,8 & 112,3 & 463,6 & 115,9 & $+8,6$ & $+28,6$ \\
\hline 9.h.A. & 50 & 121,6 & 137,0 & 112,9 & 130,9 & 502,4 & 125,6 & +9.7 & $+39,4$ \\
\hline $\begin{array}{l}10.77 \text {. } \\
6 \text { h. Vm. }\end{array}$ & 59 & 121,6 & 144,2 & 119,1 & 126,5 & 511,4 & 127,8 & $+2,2$ & $+41,8$ \\
\hline $\begin{array}{c}\text { 7. h. A. } \\
11 . / 7 .\end{array}$ & 72 & 132,6 & 150,7 & 125,9 & 129,6 & 538,8 & 134,7 & $+6,9$ & $+\quad 49,5$ \\
\hline 6. h. Vm. & 83 & 139,6 & 162,3 & 133,9 & 137,8 & 572,7 & 143,1 & $+8,4$ & $+58,8$ \\
\hline $\begin{array}{l}\text { 8. h. A. } \\
12 . / 7 .\end{array}$ & 97 & 147,0 & 174,3 & 124,0 & 145,1 & 590,4 & 147,6 & $+4,5$ & $+63,8$ \\
\hline 6. h. Vm. & 107 & 152,6 & 174,7 & 144,2 & 146,4 & 617,9 & 154,4 & $+6,8$ & $+71,3$ \\
\hline $\begin{array}{c}\text { 6. h. A. } \\
\text { 13. } / 7\end{array}$ & 119 & 162,3 & 184,8 & 144,7 & 151,3 & 643,1 & 160,7 & $+6,3$ & $+78,3$ \\
\hline 6. h. Vm. & 131 & 167,5 & 189,8 & 148,9 & 160,1 & 666,3 & 166,5 & $+5,8$ & $+84,7$ \\
\hline $\begin{array}{l}\text { 6. h. A. } \\
\text { 14./7. }\end{array}$ & 143 & 173,0 & 194,8 & 151,9 & 160,7 & 680,4 & 170,1 & $+3,6$ & $+88,7$ \\
\hline 6. h. Vm. & 155 & 179,2 & 202,9 & 161,3 & 165,6 & 709,0 & 177,2 & $+7,1$ & $+96,6$ \\
\hline 7. h. A. & 168 & 177,4 & 207,8 & 177,4 & 169,3 & 731,9 & 182,9 & $+5,7$ & $+102,9$ \\
\hline
\end{tabular}




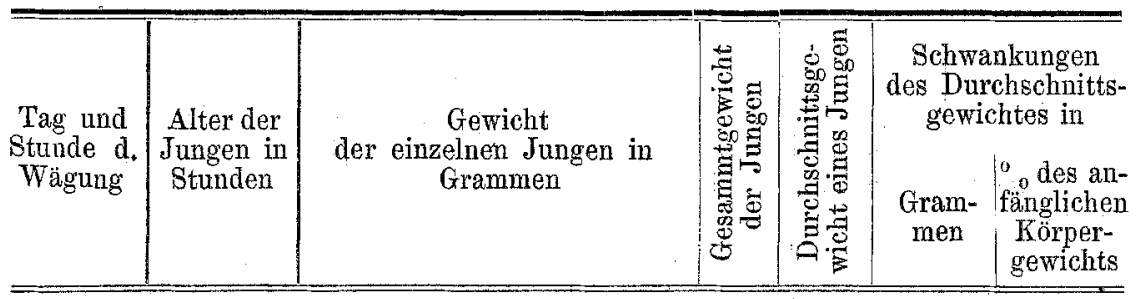

Nr. 6.

\begin{tabular}{|c|c|c|c|c|c|c|c|c|c|c|}
\hline$/ 3.1868$ & nach der & & & & & & & & & \\
\hline $\mathrm{Vm}$. & $\begin{array}{l}\text { Geburt } \mathrm{d} \text {. } \\
\text { letzten }\end{array}$ & 89,7 & 81,8 & 89,8 & 87,2 & 85,6 & 434,1 & 84,2 & - & - \\
\hline 1. h. Nm. & 3 & 94,4 & 81.8 & 91,2 & 91,8 & 87,5 & 446,7 & 89,3 & $+5,1$ & $+6,0$ \\
\hline $\begin{array}{l}8 . h . \\
25 .\end{array}$ & 10 & 96,6 & 85,6 & 95,6 & 97,8 & 96,9 & 472,5 & 94,5 & $+5,2$ & $+12,2$ \\
\hline 8. h. Vm. & 22 & 99,0 & 88,0 & 110,1 & 103,9 & 100,2 & 491,2 & 98,2 & $+3,7$ & $+16,6$ \\
\hline 1. h. & 27 & 100,2 & 90,5 & 102,4 & 104,8 & 101,0 & 498,9 & 99,7 & $+1,5$ & $+18,4$ \\
\hline $\begin{array}{l}\text { 8. h. A. } \\
26 / 3 \text {. }\end{array}$ & 34 & 101,5 & 94,1 & 106,7 & 106,8 & 101,5 & 510,6 & 102,1 & $+2,4$ & $+21,2$ \\
\hline 8. h. Vm. & 46 & 109,0 & 99,2 & 115,1 & 114,1 & 105,8 & 543,2 & 108,6 & $+6,5$ & $+28,9$ \\
\hline 1. $\mathrm{h} \mathrm{Nm}$. & 51 & 111,3 & 100,2 & 115,9 & 117,2 & 107,4 & 552,0 & 110,4 & $+1,8$ & $\begin{array}{r}+31,0 \\
0\end{array}$ \\
\hline $\begin{array}{l}\text { 8.h. A. } \\
27 . / 3\end{array}$ & 58 & 113,5 & 102,3 & 119,1 & 120,0 & 109,2 & 564,1 & 112,8 & $+2,4$ & $+33,9$ \\
\hline $8 \mathrm{~h} .1$ & 70 & 120,7 & 110,8 & 126,5 & 123,2 & 114,1 & 595,3 & 119,0 & $+6,2$ & $+41,3$ \\
\hline 8.h. A. & 82 & 128,4 & 112,1 & 131,5 & 131,6 & 115,4 & 619,0 & 123,8 & $+4,8$ & $+47,1$ \\
\hline
\end{tabular}

Nr. 7.

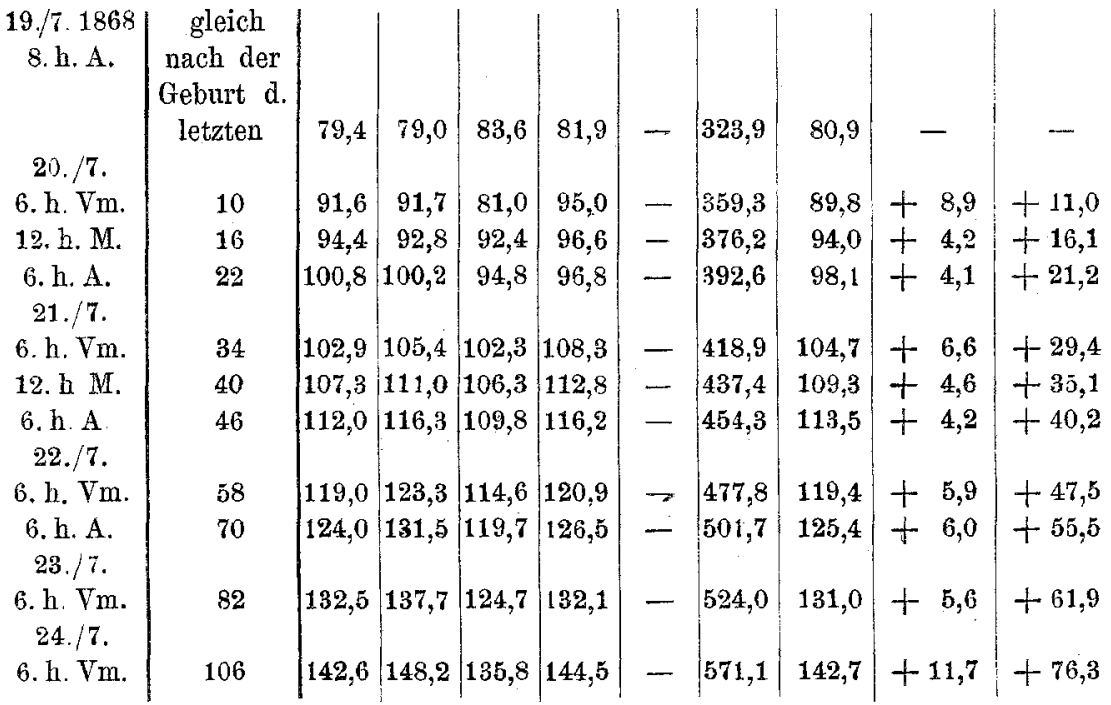




\begin{tabular}{|c|c|c|c|c|c|c|}
\hline $\begin{array}{l}\text { Tag und } \\
\text { Stunde d. } \\
\text { Wägung }\end{array}$ & $\begin{array}{l}\text { Alter der } \\
\text { Jungen in } \\
\text { Stunden }\end{array}$ & $\begin{array}{c}\text { Gewicht } \\
\text { der einzelnen Jungen in } \\
\text { Grammen }\end{array}$ & 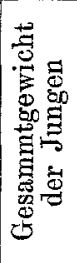 & 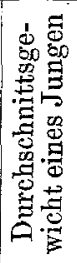 & $\begin{array}{c}\text { Schwankungen } \\
\text { des Durch- } \\
\text { schnittsgewich- } \\
\text { tes in } \\
\text { Gram- } \\
\text { men }\end{array}$ & Anmerkungen \\
\hline
\end{tabular}

Nr. 8.

\begin{tabular}{|c|c|c|c|c|c|c|c|c|c|c|}
\hline $\begin{array}{c}22 . / 8.1864 \\
\text { 7.h. Vm. }\end{array}$ & $\mid \begin{array}{c}\text { gleich } \\
\text { nach der } \\
\text { Geburt d. } \\
\text { letzten }\end{array}$ & 80,0 & 94,1 & 89,5 & 105,5 & 369,1 & 92,2 & - & - & $\begin{array}{l}\text { Mutterthier } \\
\text { an Dickdarm- } \\
\text { blenorrhoe mit } \\
\text { Chemosis ana- } \\
\text { lis leidend. }\end{array}$ \\
\hline 1. h. Nm. & 6 & 81,8 & 93,1 & 89,9 & 105,5 & 370,3 & 92,5 & $+0,3$ & $+0,3$ & \\
\hline $\begin{array}{l}\text { 9. h. A. } \\
23 . / 8 \text {. }\end{array}$ & 14 & 85,6 & 95,6 & 96,8 & 111,6 & 389,6 & 97,4 & $+4,9$ & $+5,6$ & \\
\hline 7. h. Vm. & 24 & 85,6 & 96.0 & 95,0 & 113,5 & 390,1 & 98,0 & $+0,6$ & $+6,2$ & \\
\hline 1. h. $\mathrm{Nm}$. & 30 & 90,6 & 101,7 & 101,1 & 115,4 & 408,8 & 102,2 & $+4,2$ & $+10,8$ & \\
\hline $\begin{array}{c}\text { 11. h. Nm. } \\
24 . / 8 \text {. }\end{array}$ & 40 & 92,4 & 92,4 & 107,3 & 114,1 & 406,2 & 101,5 & $-0,7$ & $+10,0$ & \\
\hline $\begin{array}{c}\text { 7. h. Vm } \\
25 . / 8\end{array}$ & 48 & 99,9 & 109,8 & 114,7 & 120,3 & 444.7 & 111,1 & $+9,6$ & $+20,5$ & \\
\hline $\begin{array}{c}\text { 7. h. Vm. } \\
26 . / 8 \text {. }\end{array}$ & 72 & 112,9 & getdt. & 127,8 & 141,4 & 382,1 & 127,3 & $+16,2$ & $+38,0$ & \\
\hline 7. h. Vm. & 96 & 117,2 & - & 130,2 & 147,6 & 395,0 & 131.6 & $+4,3$ & $+42,7$ & \\
\hline
\end{tabular}

\section{Kaninchen.}

Nr. 1.

\begin{tabular}{|c|c|c|c|c|c|c|c|c|c|c|c|}
\hline $10 . / 6.1860$ & gleich & & & & & & & & & & Das Mutter- \\
\hline 2. h. Nm. & $\begin{array}{l}\text { nach der } \\
\text { Geburt d. }\end{array}$ & & & & & & & & & & $\begin{array}{l}\text { thier wohlge- } \\
\text { nährt. }\end{array}$ \\
\hline 11. 6 . & letzten & 46,3 & $41, \tilde{5}$ & 39,9 & 44,9 & 41,1 & 213,7 & 42,7 & 一 & - & \\
\hline $\begin{array}{c}\text { 6. h. Vm. } \\
12 . / 6 .\end{array}$ & 16 & 50,6 & 46,9 & 44,6 & 49,4 & 45,9 & 237,4 & 47,4 & $+4,7$ & $+11,0$ & \\
\hline $\begin{array}{l}\text { 6. h. Vm. } \\
\text { 13./6. }\end{array}$ & 40 & 58,2 & 53,3 & 49,6 & 57,9 & 54,4 & 273,4 & 54,7 & $+7,3$ & $+28,1$ & \\
\hline $\begin{array}{c}\text { 6. h. Vm. } \\
14 . / 6 .\end{array}$ & 66 & 67,6 & 63,9 & 60,5 & 67,6 & 62,4 & 322,0 & 64,4 & $+9,7$ & $+50,8$ & \\
\hline $\begin{array}{c}\text { 6. h. Vm. } \\
\text { 15./6. }\end{array}$ & 88 & 82,9 & 77,5 & 64,5 & 80,9 & 76,9 & 382,7 & 76,5 & $+12,1$ & $+79,1$ & \\
\hline 6. h. Vm. & 112 & 96,8 & 87,2 & 79,8 & 73,2 & 92,4 & 429,4 & 85,8 & $+9,3$ & $+100,9$ & \\
\hline
\end{tabular}




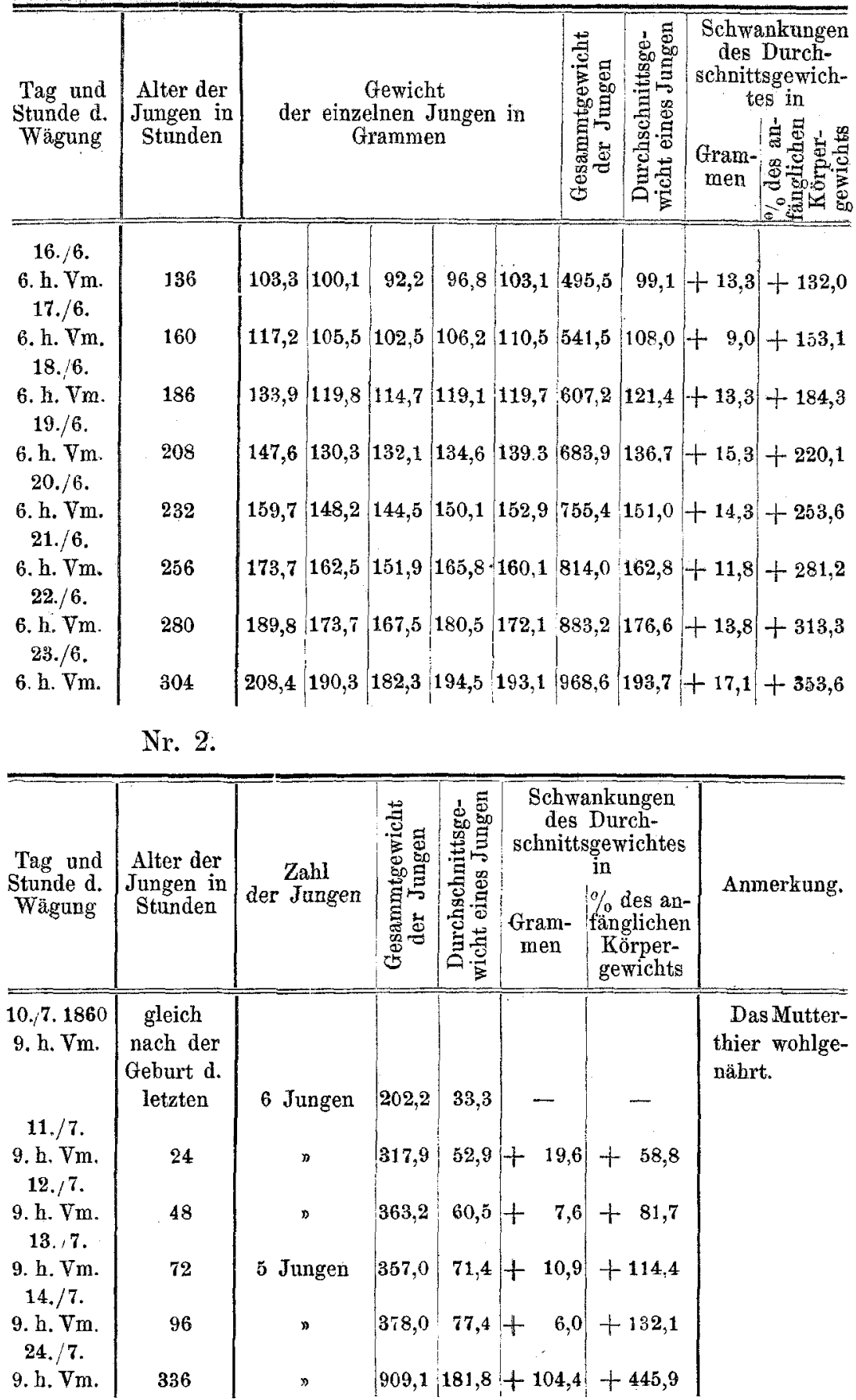




\begin{tabular}{|c|c|c|c|c|c|c|c|}
\hline $\begin{array}{l}\text { Tag und } \\
\text { Stunde d. } \\
\text { Wägung }\end{array}$ & $\begin{array}{l}\text { Alter der } \\
\text { Jungen in } \\
\text { Stunden }\end{array}$ & $\begin{array}{c}\text { Zahl } \\
\text { der Jungen }\end{array}$ & 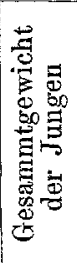 & 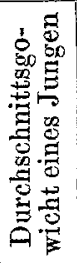 & $\begin{array}{c}\text { Schwa } \\
\text { des } \\
\text { schnitt } \\
\text { te } \\
\text { Gram- } \\
\text { men }\end{array}$ & 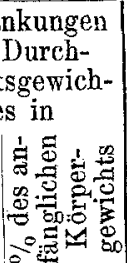 & Anmerkungen \\
\hline
\end{tabular}

Nr. 3.

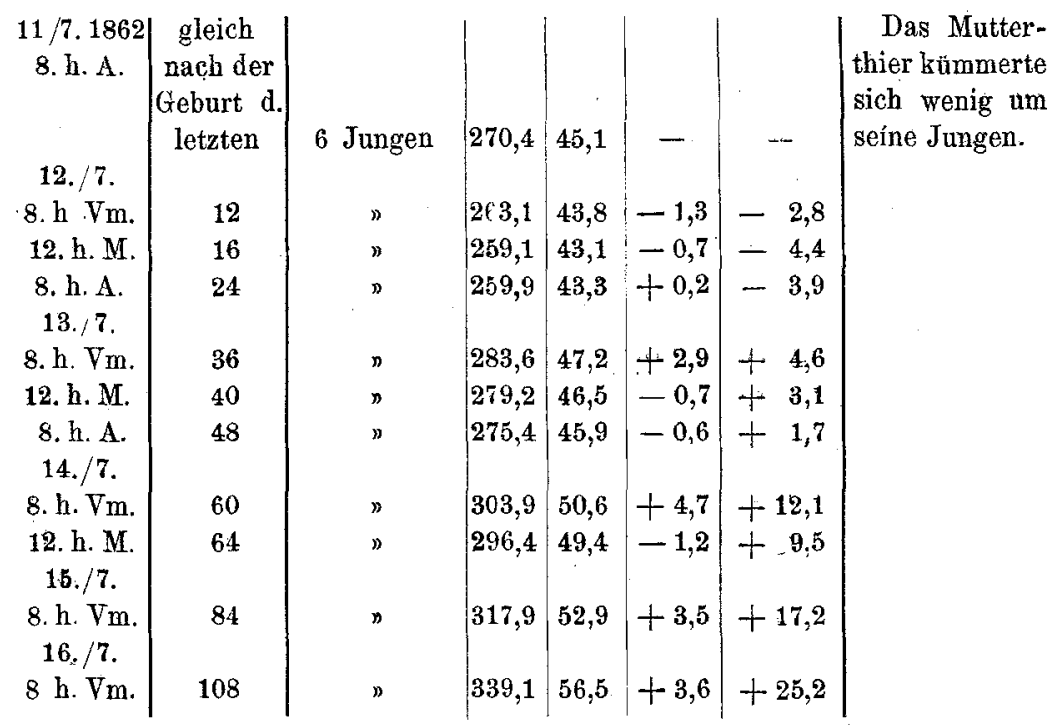

Aus den angeführten Zahlen könnte man folgern, dass die neugeborenen Säuger (zunächst die drei angeführten Species) in der Mehrzahl der Fälle, und namentlich bei genügender Ernährung der Mutterthiere, von der Geburt an fast ununterbrochen, aber ungleichmässig an Gewicht zunehmen. Dieser Schluss ist aber nicht ganz richtig. Denn auch bei dem neugeborenen Säugethiere muss die Verdunstung des an Haut und Haaren klebenden Fruchtwassers, die Entleerung von Meconium und Harn, sowie die Lungenausdünstung eine Gewichtsabnahme gleich nach der Geburt zur Folge haben. Aber diese Periode des Gewichtsverlustes dauert unter physiologischen Verhältnissen bei ihnen so kurz, wird so rasch durch Nahrungsaufnahme ausgeglichen und geht so bald in Zunahme über, dass gewöhnlich schon bei der zweiten Wägung, d. h. eine Stunde bis einen Tag nach der Geburt eine mehr min- 
der beträchtliche Gewichtsvermehrung bemerkt wurde. Man muss also genauer sagen, dass physiologischer Weise bei den genannten Säugern einer sehr kurzen Periode der Ge-wichtsabnahme eine fortschreitende Zunahme nachfolgt. Dieser rasche Eintritt der Gewichtsvermehrung ist also dem neugeborenen Säugethiere gegenüber dem .Menschen eigenthümlich und offenbart sich schon der blossen Inspection durch die fortwährend zunehmende Körperfülle, den Nichteintritt eines Collapsus wie beim Kinde.

In einer Minderzahl von Fällen wurde ein oder das andere Junge eines Wurfes, oder wurden sämmtliche Jungen einer Brut bei der zweiten oder dritten Wägung leichter gefunden als gleich. nach ihrem Austritt. Dauert aber die Abnahme fort, oder kommt nach einem vorübergehenden Ansteigen eine weitere Abnahme vor, so deutet dies entweder auf mangelhafte Ernährung oder Krankheiten der. Mutter, in Folge wovon die Milchabsonderung Störungen erleidet; auf Vernachlässigung der Jungen durch die säugende Mutter, Krankheiten der Jungen oder dergleichen Ausnahmeverhältnisse.

Betrachten wir nun die Art des Fortschreitens der Zunahme, so ist diese selbst für gleiche Zeiträume und bei blosser Berücksichtigung der Durchschnittszahlen eine sehr variable; die Schwankungen werden aber noch grösser und auffälliger, wenn man die einzelnen Individuen ins Auge fasst, und kommen hier sogar hin und wieder, selbst in.späterer Zeit, vorübergehende Gewichtsabnahmen vor. Besonders rasch und bedeutend schreitet das Wachsthum bei den jungen Kaninchen im Vergleich zu Hunden und Katzen voran.

Wie erklärt sich nun der rasche Eintritt einer Gewichtszunahme bei den neugeborenen Säugern, der späte und inconstante bei den Kindern?

Zwei Momente verdienen hier als Hauptursachen der Verschiedenheiten angeführt zu werden:

1) Die massenhafte Anhäufung von Colostrum in den Mammae zur Zeit der Geburt bei den Säugern, der späte Anfang der Milchabsonderung beim menschlichen Weibe. Wie im zweiten Hefte meiner Beiträge zur Geburtskunde S. 64 bemerkt ist, werden bei den Säugern gewöhnlich schon einige Tage vor der Geburt plötzlich viel reichlichere Mengen von Colostrum abgeschieden als in gleichen Zeiten der 
vorhergehenden Perioden der Gestation. Das Secret ist bei wohlgenährten Thieren so reichlich angehäuft, dass es oft genug schon vor der Geburt tropfenweise aus dem Euter abfliesst. Ist die Absonderung vor der Geburt spärlicher, wie z. B. bei schlecht genährten Thieren, so sehen wir doch in allen Fällen eine acute Volumenzunahme der Mammae mit reichlicher Absonderung sich einstellen, wenn die Uteruscontractionen eine grössere Energie erreichen. Während der Geburt vergrössern sich sichtlich die Euter, hauptsächlich in Folge massenhafter Milchabscheidung, wie der sofortige Collapsus nach dem Ausmelken beweist. So findet denn das neugeborene Säugethier gleich bei seinem Eintritte in die Aussenwelt eine reiche Quelle von Nahrungsmaterial, die es denn, wie wir gleich sehen werden, in ergiebigster Weise benutzt.

Anders verhält sich der zeitliche Eintritt der Milchsecretion beim menschlichen Weibe. Zwar können wir hier schon in den letzten Schwangerschaftsperioden Colostrum auspressen, und fliesst dies oft genug spontan in den letzten Schwangerschaftswochen aus. Aber der Eintritt der Geburtswehen hat keine acuite Schwellung der Brüste, keine acute Steigerung der Milchabsonderung zu Folge. Im Gegentheil dauert es $1-5$, gewölinlich drei Tage, ehe eine Intumescenz zu Stande kommt und reichlichere, für die Ernährung des Neugeborenen ausreichende Colostrummengen ausgeschieden werden.

Diese für das Wachsthum der Neugeborenen so bedeutungsvollen Unterschiede sind offenbar generelle, durch Verschiedenheiten in der Lage und dem Baue der Mammae, und ungleich innigere reflectorische Beziehungen zwischen Genital- und Brustdrüsennerven bei Mensch and Säugethier bedingt, sie sind nicht etwa als ein Symptom einer mit der Cultur zunehmenden Degeneration des Menschengeschlechtes aufzufassen, denn man beobachtet eine im Vergleich zu den Säugern späte Schwellung. und Secretion der Brüste auch bei durchaus gesunden, unter möglichst natïrchen Verhältnissen lebenden Frauen. Aber es tritt die Secretion bei gesunden kräftigen Frauen im Durchschnitt früher und energischer ein als bei schwächlichen und kranken Individuen, und ferner wirkt gerade wie bei den Säugern die Art der Ernährung in der letzten Schwangerschaftsperiode und den ersten Tagen des Wochenbettes mitbestimmend auf den Zeitpunkt des Eintrittes einer reichlichen Milchabsonderung. Und hierin scheint mir der praktische Schwerpunkt der ganzen Frage zu liegen. 
Beobachten wir die Säugethiere in ihrer natürlichen Lebensweise zu der angegebenen Periode, so sehen wir, dass sie vor und nach der Geburt in so reichlicher Menge Nahrung aufnehmen wie sonst, ja sie entwickeln gleich nach dem Gebäract. gewöhnlich einen sehr lebhaften Appetit. Unseren Hausthieren pflegt man vor dem Werfen das Futter in der sonst üblichen Menge und Beschaffenheit zu geben, aber nach der Geburt nicht viel consistente, an unverdaulichem Rückstande reiche Nahrung, sondern nahrhaftes Getränk, zu reichen, da es eine alte Erfahrung ist, dass solche frisch entbundene Thiere bei viel grober Nahrung gern Obstruction bekommen und zuweilen daran zu Grunde gehen. Aber eine kräftige Nahrung, bei den Grasfressern aus Kleie, Oelkuchen, Kartoffeln und wohl auch dem eigenen überschüssigen Colostrum, von Milch und Fleisch bei dea Carnivoren, vertragen die Thiere recht wohl und befinden sich gleich den Jungen ganz gut dabei.

Ganz anders pflegt man bekanntlich die Diät der Wöchnerinnen einzurichten. Zwar wurden von den alten griechischen Aerzten die Neuentbundenen mit kräftigen Brühen, bei den alten Deutschen mit Warmbier, Brod und Zucker gelabt, von den Franzosen mit Chaudeau aus Wein, Eigelb und Zimmt genährt und von den Engländern wird eine noch kräftigere Wochenbettskost empfohlen. Aber die Praxis vieler deutschen Gebärhäuser hat in die Verköstigung der Wöchnerinnen eine Milde und Substanzlosigkeit eingefuhrt, die zwar für das Budget vortheilhaft, aber für die Frauen selbst, und namentlich für deren Kinder nicht sehr erspriesslich sein möchte. Ein Blick in diese lauteren Fleischbrijhen, mageren Suppen, verdünnten Kaffees und Milchspeisen genügt, um auch ohne chemische Analyse den Nährwerth. dieser Diät der ersten Tage richtig zu würdigen und sich zu überzeugen, dass es sich hier oft um wenig mehr als um Zufuhr reichlichen Wassers ,mit Umständen" handelt. Es ist nun gewiss, dass die peristaltische Bewegung des Darmkanals, wahrscheinlich, dass auch die Secretion der Verdauungssäfte in den ersten Wochenbettstagen herabgesetzt ist, und es ist eine alte Erfahrung, dass aus diesem Grunde eine reichlichere Ernährung mit substanziösen Nahrungsmitteln bei der Nenentbundenen viel leichter als in anderen Lebensperioden eine Indigestion herbeiführt. Aber ebenso gewiss ist es, und die Beobachtungen in der Privatpraxis lehren dies zur Genüge, dass die Wöchnerinnen eine weit nahrhaftere, wenn auch rorzugsweise fliissige Nahrung verdauen, als ihnen in unseren Entbindungsanstalten gereicht zu werden plegt. Man 
bedenke, dass die Wöchnerinnen in den ersten neun Tagen des Wochenbettes $1 / 12$ des Körpergewichtes durch die verschiedenen Ausscheidungen verlieren, also wohl einen Anspruch haben auf baldige Restauration des Verlorenen, und dass andererseits die bei kräftigerer Diät durchschnittlich frühzeitiger eintretende Milchsecretion auch eine frühzeitigere und ergiebigere Körpergewichtszunahme des Neugeborenen zur Folge haben wird. Hieraus erwächst, wie mir scheint, die Nothwendigkeit, durch systematisch durchgeführte Beobachtungsreihen, wie sie natürlich nur in Entbindungsanstalten durchführbar sinả, festzustellen, wie grosse Mengen der verschiedenen, namentlich flüssigen Nahrungsmittel von höherem Nährwerthe man einer Wöchnerin ohne Schaden in den ersten Tagen geben kann? Sobald aber diese Menge und Qualität auf Grund umfassender Versuche mit Rücksicht auf die Constitution und den Verlauf der vorausgegangenen Geburt für die einzelnen Wochenbetttage u. s. w. festgestellt ist, wird sich die fernere Nothwendigkeit ergeben, die erlaubte Nahrung zur Verhütung eines allzugrossen Collapsus der Mutter wie des Kindes in der That zu reichen, und sich nicht immerfort, zum grossen Nachtheile der Kinder, mit Abschlagsportionen zu begnügen.

2) Eine zweite Ursache des ungleich raschen Eintrittes der Körpergewichtszunahme beim neugeborenen Kinde und Säugethiere liegt in dem Verhalten der Neugeborenen selbst.

Die Jungen der zu unseren Wägungen benutzten kleineren Säuger (Hunde, Katzen, Kaninchen) suchen, trotz ihrer anfänglichen Blindheit, blos von einem lebhaft entwickelten Tastgefühle geleitet, sofort nach ihrer Geburt, oft noch am Nabelstrange hängend, die mütterlichen Warzen auf und beginnen nun ein prolongirtes Saugen, das in der ersten Zeit nur vorübergehend durch Schlafen unterbrochen wird. Wenn auch nicht ebensobald, so beginnen doch schon innerhalb der ersten Stunden nach der Geburt die Jungen anderer Säuger das Saugen, am frühesten unter den Hausthieren das Schwein, welches zunächst durch Zug und nöthigenfalls durch Druck mit den Hinterklauen den Nabelstrang zerreisst und, zur Ortsbewegung sofort befähigt, zu dem Euter eilt; meist innerhalb einer Stunde das Schaaf und die Ziege, etwas später Fohlen und Kalb - Wiederkäuer und Einhufer deshalb später, weil sie das Aufrechtstehen erst nach mehreren erfolglosen Versuchen erlernen. 
Wie hülflos und imbecil, erscheint den jungen Säugethieren gegenüber das neugeborene Kind! Die Fähigkeit der Ortsbewegung geht ihm vollständig ab, und wenn auch die kräftigeren Kinder schon im Verlaufe des ersten Tages durch Bewegungen der Lippen ihr Nahrungsbedürfniss zu verrathen scheinen und die dargebotene Warze fassen und ansaugen, so dauert es bei Anderen 2-3 Tage, ehe sie gehörig zu saugen vermögen.

Den beiden genannten Momenten gegenüber treten die übrigen Ursachen der Unterschiede der Gewichtsveränderungen der neugeborenen Kinder und Säugethiere unstreitig in den Hintergrund, denn die Ausscheidungen von Meconium und Harn, Perspirations - und Respirationsgasen geschehen bei allen Neugeborenen und sind relativ zum Körpergewicht nicht so verschieden, um auch nur annähernd die Differenzen der Körpergewichtsschwankungen erklären zu können. Wohl mag das Kind zur Erzeugung der nöthigen Wärmemengen einen lebhafteren Stoffwechsel nöthig haben als die Jungen derjenigen Species, welche gleichsam abortiv geboren werden und nicht blos durch Aufenthalt in einem Neste und gegenseitige Erwärmung, sondern auch durch die Wärmezufuhr von Seiten des sie deckenden Mutterthieres geringere Wärmeverluste haben. Aber andererseits sehen wir, dass bei vielen Säugern die Jungen gegen strahlende Wärme nur durch Haare u. dergl., nicht aber durch accessorische Mittel geschützt sind, und doch rasch nach der Geburt an Gewicht zuzunehmen anfangen, zum Beweis, dass die zur Zeit der Geburt reichlich vorhandene Mich sowie die baldige Befähigung der Neugeborenen zun Saugen die Hauptursache des geringen Gewichtsabfalles und der raschen Gewichtszunahme abgeben.

Die Wägungen neugeborener bloss mit Muttermilch ernährter Thiere, sowie die Beobachtung ihres zunehmenden Embonpoint zeigen wohl zur Genüge, dass die verbreitete Vorstellung von dem geringen Nährwerthe und der vorzugsweise abführenden Wirkung des Colostrum unbegründet ist.

Fassen wir die soeben gewonnenen Ergebnisse der vergleichenden Untersuchung noch einen Augenblick rom praktischen Standpunkte aus ins Auge, so ergeben sich einige wichtige Folgerungen.

Die Eineist die, dass wir das Neugeborene möglichst bald an die Brust geben, theils um es zur frühzeitigen Uebung der Saugbewegungen zu bestimmen, theils auch um durch Reizung der War- 
zennerven eine baldige reflectorische Congestion und Secretion der Mammae anzuregen, - eine längst in der Praxis beobachtete Regel, deren Bedeutung sofort einleuchtet.

Weit entfernt zu glauben, dasss eine kräftige Ernährung der Hochschwangeren und Neuentbundenen eine so frühzeitige und reichliche Milchabsonderung veranlassen werde, um dadurch die primäre Gewichtsabnahme der Neugeborenen zu verhüten, möchte ich doch darauf hinweisen, dass die Einführung einer nahrhafteren, leicht resorbirbaren Nahrung in die Diät auch der ersten Wochenbettstage - natürlich mit Ausnahme der Zeit des Milchfiebers - durch Verkürzung der Periode des Gewichtsverlustes dem Neugeborenen von grossem Vortheil sein möchte. Gewiss ist es für dessen weitere Entwickelung von hoher Bedeutung, wie bedeutend das Gewicht sich vermindert und wie lange der Collapsus dauert, denn leicht erreicht diese physiologische Atrophie eine pathologische Höhe, die nur zu oft mit dem Exitus lethalis abschliesst. Nähren wir unsere Wöchnerinnen besser an den Kindern wird es sich lohnen! 